\title{
Successful laparoscopic surgery for 14-month-old infant with ovarian torsion
}

\author{
Ji Hyun Ahn', Jaeyeon Chung', Taek Sang Lee ${ }^{1,2}$ \\ Department of Obstetrics and Gynecology, ${ }^{1}$ Seoul National University College of Medicine, ${ }^{2}$ SMG-SNU Boramae Medical Center, Seoul, Korea
}

The prevalence of ovarian torsion is 4.9 among 100,000 females between ages 1 to 20 years. The diagnosis of ovarian torsion in children, especially in infants, is very difficult. Since they cannot explain related symptoms accurately, and reproductive organs lie high in the abdomen, physical examination shows unclear findings. For these reasons, we use imaging studies, such as ultrasonography and magnetic resonance imaging, to diagnose ovarian torsion. However, it is of limited value to diagnose by using these modalities alone. Therefore, clinical suspicion is important for diagnosis. Though pediatric laparoscopic surgery was introduced 20 years ago, it has been widely performed since the mid 1990s with the development of 3-mm instruments. In addition, usually the pediatric operation is done in the pediatric surgery office, even though it is a gynecologic procedure. In addition, laparotomy is still more frequently conducted in current clinical practice, although the frequency of laparoscopic surgery has increased. However, it is thought that expert gynecologic surgeons can perform pediatric laparoscopic operations if they pay attention to some precautions. We report herein the case of a 14-month-old infant who underwent emergency laparoscopic untwisting of ovarian torsion successfully without complications by a gynecologic surgeon, with a brief review of the literature.

Keywords: Infant; Laparoscopy; Ovarian torsion

\section{Introduction}

The past 4 decades have witnessed rapid progress and technologic advances in gynecologic laparoscopy, which have made most procedures feasible through laparotomy. Although the feasibility of laparoscopic surgery has not been well established in infants due to the small abdominal cavity and limited view [1], diagnostic laparoscopy has been regarded as one of the best diagnostic modalities because symptoms related to pelvic masses are not specific, and preoperative diagnosis depends on the exclusion of relevant disease despite technologic advances in imaging [2].

Here, we introduce the case of a 14-month-old infant with ovarian torsion, who underwent successful laparoscopic surgery by a gynecologic surgeon, and discuss the feasibility and special considerations of laparoscopic surgery in infants with a brief literature review.

\section{Case report}

A 14-month-old girl weighing $10.4 \mathrm{~kg}$ visited our clinic with a 3-day history of irritability and vomiting. She have had no special medical history and other symptoms except for poor oral intake prior to this presentation. Furthermore, there were no abnormal findings in physical examination or laboratory tests except that her white blood cell count was within the upper limit (11,760/up; normal range, 4,000-1,2000/up). Transabdominal ultrasonography (USG) showed the enlarged right adnexa suggestive of ovarian torsion (Fig. 1A). Magnetic resonance imaging (MRI) for pelvic organs revealed an

Received: 2013.8.12. Revised: 2013.9.17. Accepted: 2013.9.21.

Corresponding author: Taek Sang Lee

Department of Obstetrics and Gynecology, SMG-SNU Boramae Medical Center, 20 Boramae-ro 5-gil, Dongjak-gu, Seoul 156-707, Korea

Tel: +82-2-870-2346 Fax: +82-2-831-2826

E-mail: tslee70@gmail.com

Articles published in Obstet Gynecol Sci are open-access, distributed under the terms of the Creative Commons Attribution Non-Commercial License (http://creativecommons. org/licenses/by-nc/3.0/) which permits unrestricted non-commercial use, distribution, and reproduction in any medium, provided the original work is properly cited.

Copyright $\odot 2014$ Korean Society of Obstetrics and Gynecology 


\section{Obstetrics \& Gynecology Science}

Ji Hyun Ahn, et al. Laparoscopic surgery for infant is safe
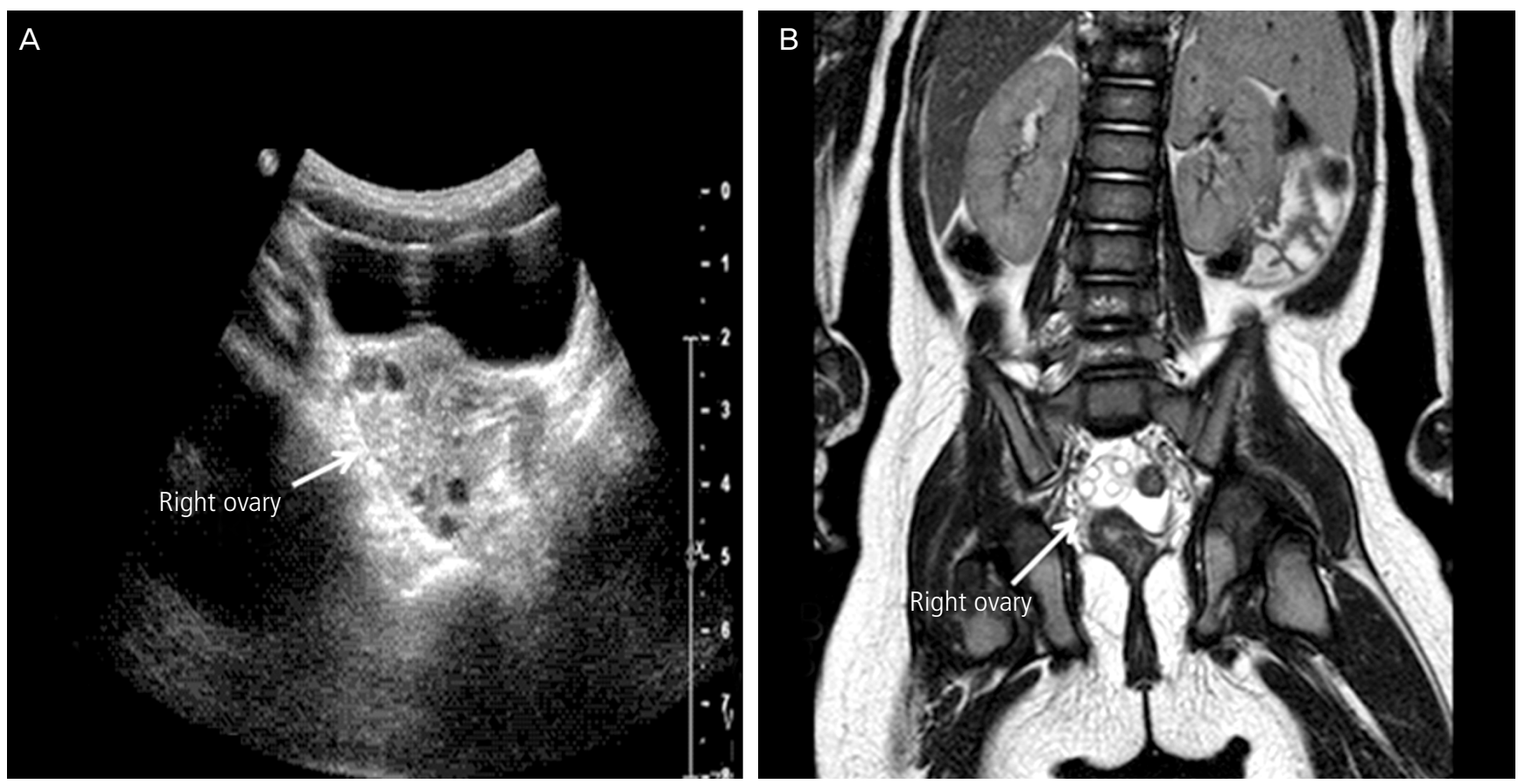

Fig. 1. (A) Enlarged right ovary in ultrasonography. (B) Edematous ovary with decreased enhancement in magnetic resonance imaging.
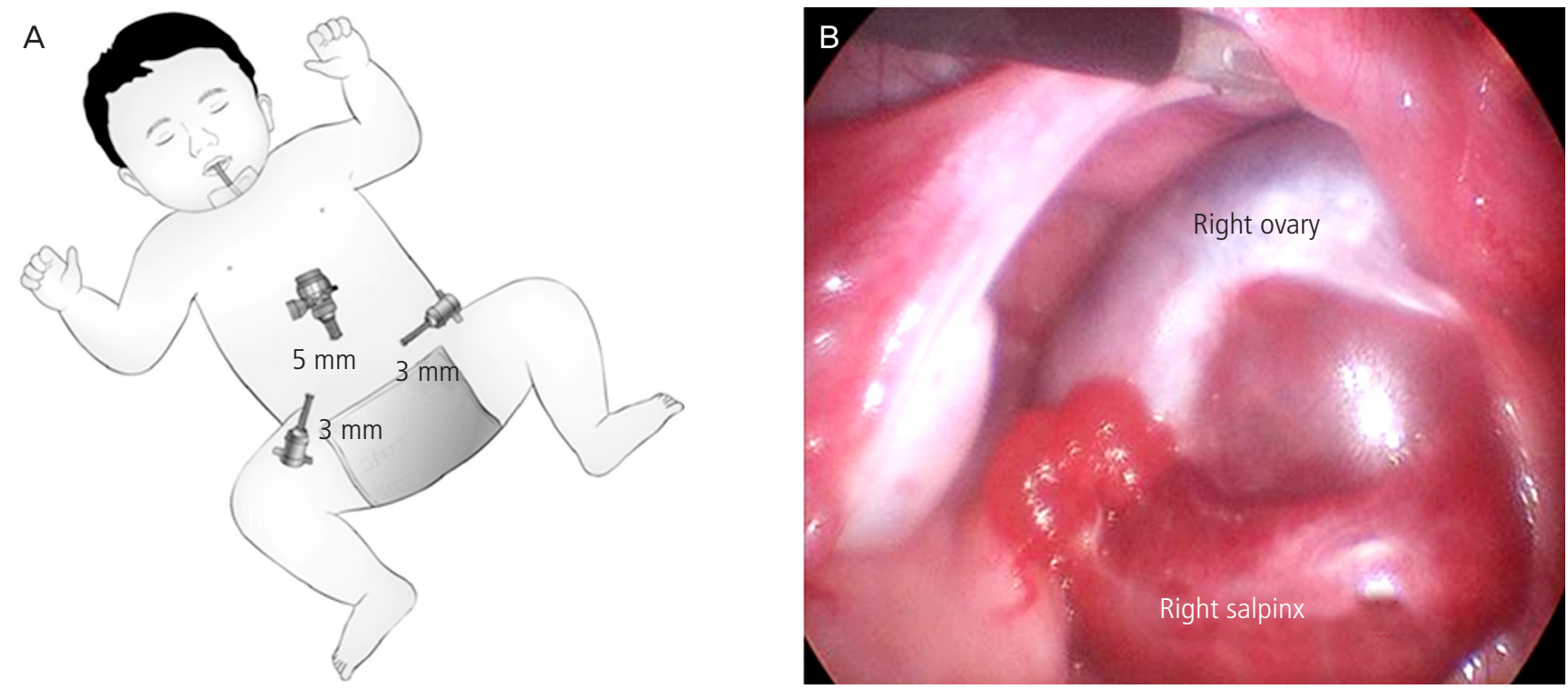

Fig. 2. (A) Intra-operation position and trocar insertion. (B) Edematous change of the right fallopian tube.

edematous right ovary that was enlarged to $3.6 \mathrm{~cm}$ with decreased enhancement (Fig. 1B). Thus, we planned to perform an emergent laparoscopic surgery for confirmative diagnosis and surgical treatment.

After insufflation using a Verres needle, we inserted 5-mm diameter cannulas into the umbilicus, and two 3-mm ancil- lary cannulas were inserted into both lower quadrants of the abdomen (Fig. 2A). During laparoscopy, abdominal pressure was maintained below $8 \mathrm{mmHg}$, and the edematous change of the right fallopian tube was found because the tube was twisted without knot formation or ovarian cysts (Fig. 2B). Thus, we only untwisted the right adnexa using 3-mm diam- 


\title{
Obstetrics \& Gynecology Science
}

\author{
Vol. 57, No. 2, 2014
}

eter forceps, and completed all procedures without complications or bleeding. The operating time was 25 minutes, and her irritability decreased after surgery. She was discharged from the hospital 2 days after surgery. There was no abnormal finding when she visited our department 10 days after surgery.

\section{Discussion}

Since maternal hormones stimulate fetal ovaries, cystic changes can be seen in one-third of all female infants [3]. As a result, the estimated incidence of ovarian torsion is 4.9 among 100,000 females with ages 1 to 20 years [4], and ovarian torsion accounts for up to $2.7 \%$ of all children with acute abdominal pain [5]. In particular, ovarian cysts $5 \mathrm{~cm}$ in diameter have a higher incidence of complications, such as rupture, bleeding, or torsion, and the risk of ovarian torsion increases by $50 \%$ to $78 \%$ in infants [6]. Although an underlying cyst is the most common cause of ovarian torsion in adults, the etiology remains unclear in half of the infants or children $[4,7]$. In infants, the length of fallopian tubes or suspensory ligaments can influence ovarian mobility and subsequent vascular compromise without ovarian cystic lesions. In this case, we also found that the right fallopian tube twisted without ovarian cystic lesions.

However, the diagnosis of ovarian torsion is very difficult in pediatric patients because the children cannot explain related symptoms accurately, and reproductive organs lie high in the abdomen, causing unclear physical examination findings. Furthermore, physicians should be aware of other causes of acute abdomen, including appendicitis, urinary tract infection and gastroenteritis [8]. Thus, imaging studies, such as USG and MRI, are helpful in preoperative diagnosis of ovarian torsion. First, doppler USG has an important value as a diagnostic tool because of its high specificity. Nevertheless, it shows a low sensitivity of $60 \%$ due to partial intermittent torsion or dual blood supply from the ovarian arteries, which means that visualization of blood flow on doppler USG cannot exclude ovarian torsion [9]. Second, MRI may be more valuable in detecting a thickened tubes visualized as a tubular protrusion on the twisted side [10]. However, ovarian torsion still remains a challenge requiring clinical diagnosis because imaging studies are not reliable to confirm it.

For the treatment, advances in technology and surgical expertise have extended the use of laparoscopic surgery to infants because the infants tolerate pneumoperitoneum well. Laparoscopy not only provides a magnified view of the entire abdomen, including both ovaries but also allows therapeutic interventions, such as cyst aspiration or excision, and detorsion to minimize surgical morbidity $[11,12]$. In particular, a trend towards a more conservative treatment approach has emerged over the past few years, and laparoscopic detorsion is the current choice of treatment for ovarian torsion in pediatric patients. Although the danger of missing malignant pathology, the risk of thromboembolic complications and the fear that a severe ischemic ovary is non-viable sometimes lead to oophorectomy or salingo-oophorectomy, recent studies show a low risk of malignancy, and thromboembolism associated with ovarian torsion, and that the macroscopic appearance of ovary is not a true indicator of the degree of ischemia, encouraging more conservative surgical treatment, especially, in children $[5,13]$.

For the safety of laparoscopic surgery, relevant studies have suggested some precautions for infants as follows. First, videoguided insertion of the first cannula is safe to avoid damage to organs within the small abdominal cavity. Second, it is usually possible to use trocars or cannulas with a diameter of $1.7,3$, or $5 \mathrm{~mm}$ for less invasive surgery. Third, no trocar should be placed through the umbilicus during the first month of life because it may cause still patent umbilical vessels to bleed. Fourth, intra-abdominal pressure should not exceed 8 $\mathrm{mmHg}$, and lengthy interventions should also be avoided because of the risk of desaturation during laparoscopic surgery $[11,12,14]$. When infants have ovarian cystic lesions with torsion, some physicians prefer percutaneous needle aspiration as less invasive procedure in spite of the feasibility of laparoscopy. However, this procedure should be cautiously performed in infants because other diseases, such as small bowel duplication, may sometimes be misdiagnosed as ovarian torsion, which can be lethal if cyst aspiration is performed [15].

In conclusion, the results of this study suggest that laparoscopic surgery can be performed in infants with gynecologic diseases and that less invasive techniques, such as the use of 3-mm diameter cannulas, could be applied to infants when underlying disease has been resolved by simple procedures, such as untwisting.

It is thought that expert gynecologic surgeons can perform pediatric laparoscopic operation successfully if they pay attention to the aforementioned precautions. 


\section{Obstetrics \& Gynecology Science}

Ji Hyun Ahn, et al. Laparoscopic surgery for infant is safe

\section{Conflict of interest}

No potential conflict of interest relevant to this article was reported.

\section{References}

1. Ozcan R, Kuruoglu S, Derviaoglu S, Elicevik M, Emir H, Buyukunal $C$. Ovary-sparing surgery for teratomas in children. Pediatr Surg Int 2013;29:233-7.

2. Oltmann SC, Fischer A, Barber R, Huang R, Hicks B, Garcia N. Cannot exclude torsion: a 15-year review. J Pediatr Surg 2009;44:1212-6.

3. Luzzatto C, Midrio P, Toffolutti T, Suma V. Neonatal ovarian cysts: management and follow-up. Pediatr Surg Int 2000;16:56-9.

4. Gocmen A, Karaca M, Sari A. Conservative laparoscopic approach to adnexal torsion. Arch Gynecol Obstet 2008;277:535-8.

5. Aziz D, Davis V, Allen L, Langer JC. Ovarian torsion in children: is oophorectomy necessary? J Pediatr Surg 2004;39:750-3.

6. Bagolan P, Giorlandino C, Nahom A, Bilancioni E, Trucchi A, Gatti $C$, et al. The management of fetal ovarian cysts. J Pediatr Surg 2002;37:25-30.

7. Houry D, Abbott JT. Ovarian torsion: a fifteen-year review. Ann Emerg Med 2001;38:156-9.

8. Poonai N, Poonai C, Lim R, Lynch T. Pediatric ovarian tor- sion: case series and review of the literature. Can J Surg 2013; 56:103-8.

9. Chiou SY, Lev-Toaff AS, Masuda E, Feld RI, Bergin D. Adnexal torsion: new clinical and imaging observations by sonography, computed tomography, and magnetic resonance imaging. J Ultrasound Med 2007;26:1289-301.

10. Rha SE, Byun JY, Jung SE, Jung JI, Choi BG, Kim BS, et al. $\mathrm{CT}$ and MR imaging features of adnexal torsion. Radiographics 2002;22:283-94.

11. Esposito C, Garipoli V, Di Matteo G, De Pasquale M. Laparoscopic management of ovarian cysts in newborns. Surg Endosc 1998;12:1152-4.

12. Van der Zee DC, van Seumeren IG, Bax KM, Rovekamp $\mathrm{MH}$, ter Gunne AJ. Laparoscopic approach to surgical management of ovarian cysts in the newborn. J Pediatr Surg 1995;30:42-3.

13. Katara AN, Shah RS, Bhandarkar DS, Shaikh S. Laparoscopic management of antenatally-diagnosed abdominal cysts in newborns. Surg Laparosc Endosc Percutan Tech 2004;14:42-4.

14. Spinelli C, Buti I, Pucci V, Liserre J, Alberti E, Nencini $L$, et al. Adnexal torsion in children and adolescents: new trends to conservative surgical approach: our experience and review of literature. Gynecol Endocrinol 2013;29:54-8.

15. Puligandla PS, Laberge JM. Lethal outcome after percutaneous aspiration of a presumed ovarian cyst in a neonate. Semin Pediatr Surg 2009;18:119-21. 